\title{
FRACTIONAL INTEGRAL OPERATORS INVOLVING THE PRODUCT OF SRIVASTAVA POLYNOMIALS AND SRIVASTAVA-PANDA MULTIVARIABLE $H$-FUNCTIONS OF GENERALIZED ARGUMENTS
}

\author{
V. B. L. CHAURASIA AND S. C. PANDEY
}

\begin{abstract}
The paper deals with two fractional integral formulae involving the product of a general class of polynomials and multivariate $H$-function. The first involves the operator ${ }_{c} I_{z}^{v}[f(z)]$ whereas second is associated with the integral operator $I_{z}^{\eta, v}[f(z)]$. In our fractional integral formulae we have taken all the functions and polynomials with a generalized argument. The formulae, we have introduced here, are in compact form and basic in nature. A number of known and new results have been obtained by proper choice of parameters. For the sake of illustration, we record here some particular cases of our main results.
\end{abstract}

\section{Introduction}

In recent years several authors (see, for example) Chen et al. [13], Lin et al. [16], Soni et al. [15], seee also Gaira et al. [12] have made significant contributions to the fractional calculus operators involving various functions and polynomials. Here we are making an attempt to develop extensions of these results.

We start by introducing following definitions:

Oldham and Spanier [8] considered the fractional integral of a function $f(z)$ of complex order $v$

$$
{ }_{c} I_{z}^{v}[f(z)]=\left\{\begin{array}{l}
\frac{1}{\Gamma v} \int_{c}^{z}(z-t)^{\nu-1} f(t) d t, \quad \operatorname{Re}(v)>0 \\
\frac{d^{q}}{d z^{q}} c I_{z}^{\nu+q}[f(z)], \quad \operatorname{Re}(\nu) \leq 0,0<\operatorname{Re}(v)+q \leq 1, \quad q=1,2,3, \ldots
\end{array}\right.
$$

The special case of fractional integral operator ${ }_{c} I_{z}^{v}$, when $c=0$, will be denoted by $I_{z}^{v}$. Thus we write

$$
I_{z}^{v} \equiv{ }_{0} I_{z}^{v}
$$

and

$$
I_{z}^{\nu}[f(z)]=\left\{\begin{array}{l}
\frac{1}{\Gamma v} \int_{c}^{z}(z-t)^{\nu-1} f(t) d t, \operatorname{Re}(v)>0 \\
\frac{d^{q}}{d z^{q}} I_{z}^{\nu+q}[f(z)], \quad \operatorname{Re}(v) \leq 0,0<\operatorname{Re}(v)+q \leq 1, \quad q=1,2,3, \ldots
\end{array}\right.
$$

Received November 14, 2006; revised May 24, 2007.

2000 Mathematics Subject Classification. 26A33, 33C70.

Key words and phrases. Fractional integral operators, general class of polynomials, multivariable $\mathrm{H}$ function. 
The above integral operator $I_{z}^{v}[f(z)]$ is called Riemann-Liouville fractional integral operator.

Also, Srivastava [3] introduced general calss of polynomials

$$
S_{n}^{m}[x]=\sum_{k=0}^{[n / m]} \frac{(-n)_{m k}}{k !} A_{n, k} x^{k}, \quad n=0,1, \ldots
$$

where $m$ is an arbitrary positive integer and coefficients $A_{n, k}(n, k \geq 0)$ are arbitrary constants, real or complex. On suitably specializing the coefficients $A_{n, k}, S_{n}^{m}[x]$ yields a number of known polynomials as its special cases ([14], p.158-161).

The $H$-function of several complex variables [3] is defined in the following manner:

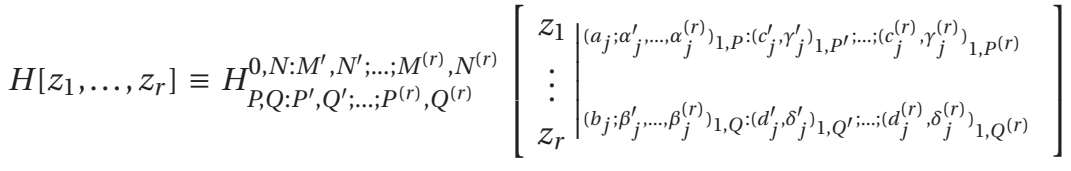

$$
\begin{aligned}
& =\frac{1}{2 \pi \omega} \int_{L_{1}} \cdots \int_{L_{r}} \phi_{1}\left(\xi_{1}\right) \cdots \phi_{r}\left(\xi_{r}\right) \psi\left(\xi_{1}, \ldots, \xi_{r}\right) z_{1}^{\xi_{1}} \cdots z_{r}^{\xi_{r}} d \xi_{1} \cdots d \xi_{r}
\end{aligned}
$$

where $\omega=\sqrt{-1}$

$$
\begin{gathered}
\phi_{i}\left(\xi_{i}\right)=\frac{\prod_{j=1}^{M^{(i)}} \Gamma\left(d_{j}^{(i)}-\delta_{j}^{(i)} \xi_{i}\right) \prod_{j=1}^{N^{(i)}} \Gamma\left(1-c_{j}^{(i)}+\gamma_{j}^{(i)} \xi_{i}\right)}{\prod_{M^{(i)}+1}^{Q^{(i)}} \Gamma\left(1-d_{j}^{(i)}+\delta_{j}^{(i)} \xi_{i}\right) \prod_{j=N^{(i)}+1}^{P^{(i)}} \Gamma\left(c_{j}^{(i)}-\gamma_{j}^{(i)} \xi_{i}\right)} \quad \forall i \in\{1, \ldots, r\} \\
\text { and } \psi\left(\xi_{1}, \ldots, \xi_{r}\right)=\frac{\prod_{j=1}^{N} \Gamma\left(1-a_{j}+\sum_{i=1}^{r} \alpha_{j}^{(i)} \xi_{i}\right)}{\prod_{j=N+1}^{P} \Gamma\left(a_{j}-\sum_{i=1}^{r} \alpha_{j}^{(i)} \xi_{i}\right) \prod_{j=1}^{Q}\left(1-b_{j}+\sum_{i=1}^{r} \beta_{j}^{(i)} \xi_{i}\right)} .
\end{gathered}
$$

The convergence conditions and other details of the above function are given by Srivastava, Gupta and Goyal ([4], p.251, eq. (c.1), also see P.252-253, eq. (c.5 and c.6)). 


\section{Main results}

\section{A. Fractional integral formula 1}

$$
\begin{aligned}
& { }_{c} I_{z}^{v}\left[z^{\rho} \prod_{i=1}^{t}\left(z+\alpha_{i}\right)^{\sigma_{i}} \prod_{j=1}^{s} S_{n_{j}}^{m_{j}}\left[e_{j} z^{u_{j}} \prod_{i=1}^{t}\left(z+\alpha_{i}\right)^{u_{j}^{(i)}}\right]\right. \\
& \left.\times H\left[x_{1} z^{\mu_{1}} \prod_{i=1}^{t}\left(z+\alpha_{i}\right)^{\mu_{1}^{(i)}}, \ldots, x_{r} z^{\mu_{r}} \prod_{i=1}^{t}\left(z+\alpha_{i}\right)^{\mu_{r}^{(i)}}\right]\right] \\
& =z^{\rho}\left(\alpha_{1}\right)^{\sigma_{1}} \cdots\left(\alpha_{t}\right)^{\sigma_{t}} \sum_{g_{1}=0}^{\infty} \cdots \sum_{g_{t}=0}^{\infty} \sum_{R=0}^{\infty} \sum_{k_{1}=0}^{\left[n_{1} / m_{1}\right]} \cdots \sum_{k_{s}=0}^{\left[n_{s} / m_{s}\right]} \frac{\left(-n_{1}\right)_{m_{1} k_{1}}}{k_{1} ! g_{1} !} A_{n_{1}, k_{1}}^{(1)} \cdots \\
& \times \frac{\left(-n_{s}\right)_{m_{s} k_{s}} A_{n_{s}, k_{s}}^{(s)}}{k_{s} ! g_{s} !} e_{1}^{k_{1}} \cdots e_{s}^{k_{s}}\left(\alpha_{1}\right)^{\left[u_{1}^{1} k_{1}+\cdots+u_{s}^{1} k_{s}-g_{1}\right]} \cdots\left(\alpha_{t}\right)^{\left[u_{1}^{t} k_{1}+\cdots+u_{s}^{t} k_{s}-g_{t}\right]} \\
& \times \frac{(-1)^{R}(z-c)^{R+v}}{\Gamma(v)(R+v) R !} z^{\left[u_{1} k_{1}+\cdots+u_{s} k_{s}+g_{1}+\cdots+g_{t}-R\right]} H_{P+(t+1), Q+(t+1): P^{\prime}, Q^{\prime} ; \ldots ; P^{(r)}, Q^{(r)}}^{0, N+(t+1): M^{\prime}, N^{\prime} ; \ldots ; M^{(r)}, N^{(r)}}
\end{aligned}
$$

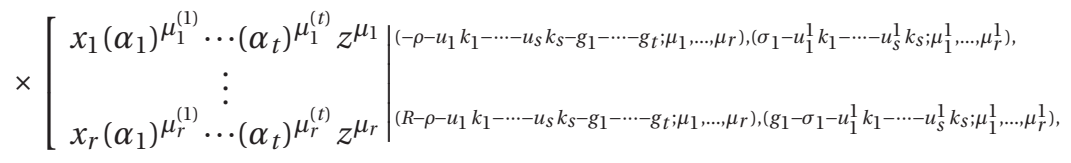

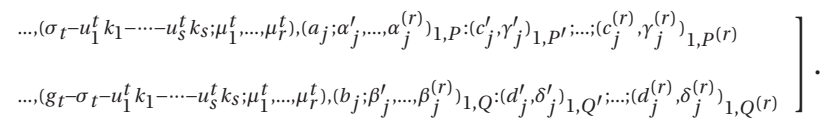

Also, by the application of following formula in (2.1)

$$
\begin{aligned}
& \sum_{R=0}^{\infty} \frac{(-1)^{R}}{\Gamma(\nu)(R+v) R !} H_{P, Q+1: P^{\prime}, Q^{\prime} ; \ldots ; P^{(r)}, Q^{(r)}}^{0, N: M^{\prime}, N^{\prime} ; \ldots ; M^{(r)}, N^{(r)}} \\
& \times\left[\begin{array}{c|cc}
z_{1} & \left(a_{j} ; \alpha_{j}^{\prime}, \ldots, \alpha_{j}^{(r)}\right)_{1, P} & :\left(c_{j}^{\prime}, \gamma_{j}^{\prime}\right)_{1, P^{\prime} ; \ldots ;\left(c_{j}^{(r)}, \gamma_{j}^{(r)}\right)_{1, P}(r)} \\
\vdots & \left(R-k ; u_{1}, \ldots, u_{r}\right),\left(b_{j} ; \beta_{j}^{\prime}, \ldots, \beta_{j}^{(r)}\right)_{1, Q}:\left(d_{j}^{\prime}, \delta_{j}^{\prime}\right)_{1, Q^{\prime} ; \ldots ;\left(d_{j}^{(r)}, \delta_{j}^{(r)}\right)_{1, Q}(r)}
\end{array}\right]
\end{aligned}
$$

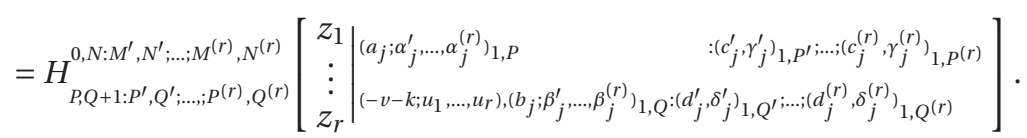

We can write

$$
\begin{aligned}
& I_{z}^{v}\left\{z^{\rho} \prod_{i=1}^{t}\left(z+\alpha_{i}\right)^{\sigma_{i}} \prod_{j=1}^{s} S_{n_{j}}^{m_{j}}\left[e_{j} z^{u_{j}} \prod_{i=1}^{t}\left(z+\alpha_{i}\right)^{u_{j}^{(i)}}\right]\right. \\
& \left.\quad \times H\left[x_{1} z^{\mu_{1}} \prod_{i=1}^{t}\left(z+\alpha_{i}\right)^{\mu_{1}^{(i)}}, \ldots, x_{r} z^{\mu_{r}} \prod_{i=1}^{t}\left(z+\alpha_{i}\right)^{\mu_{r}^{(i)}}\right]\right\} \\
& =z^{\rho+v}\left(\alpha_{1}\right)^{\sigma_{1}} \cdots\left(\alpha_{t}\right)^{\sigma_{t}} \sum_{g_{1}=0}^{\infty} \cdots \sum_{g_{t}=0}^{\infty} \sum_{k_{1}=0}^{\left[n_{1} / m_{1}\right]} \cdots \sum_{k_{s}=0}^{\left[n_{s} / m_{s}\right]} \frac{\left(-n_{1}\right)_{m_{1} k_{1}}}{k_{1} ! g_{1} !} A_{n_{1}, k_{1}}^{(1)} \cdots
\end{aligned}
$$




$$
\begin{aligned}
& \times \frac{\left(-n_{s}\right)_{m_{s} k_{s}} A_{n_{s}, k_{s}}^{(s)}}{k_{s} ! g_{s} !} e_{1}^{k_{1}} \cdots e_{s}^{k_{s}}\left(\alpha_{1}\right)^{\left[u_{1}^{1} k_{1}+\cdots+u_{s}^{1} k_{s}-g_{1}\right]} \cdots\left(\alpha_{t}\right)^{\left[u_{1}^{t} k_{1}+\cdots+u_{s}^{t} k_{s}-g_{t}\right]} \\
& \times z^{\left[u_{1} k_{1}+\cdots+u_{s} k_{s}+g_{1}+\cdots+g_{t}\right]} H_{P+(t+1), Q+(t+1): P^{\prime}, Q^{\prime} ; \ldots ; P^{(r)}, Q^{(r)}}^{0, N+(t+1): M^{\prime}, N^{\prime} ; \ldots ; M^{(r)}, N^{(r)}}
\end{aligned}
$$

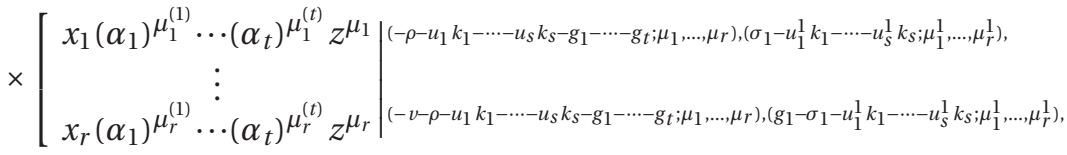

$$
\begin{aligned}
& \left.\begin{array}{l}
\ldots,\left(\sigma_{t}-u_{1}^{t} k_{1}-\cdots-u_{s}^{t} k_{s} ; \mu_{1}^{t}, \ldots, \mu_{r}^{t}\right),\left(a_{j} ; \alpha_{j}^{\prime}, \ldots, \alpha_{j}^{(r)}\right)_{1, P}:\left(c_{j}^{\prime}, \gamma_{j}^{\prime}\right)_{1, P^{\prime} ; \ldots ;\left(c_{j}^{(r)}, \gamma_{j}^{(r)}\right)_{1, P}(r)} \\
\ldots,\left(g_{t}-\sigma_{t}-u_{1}^{t} k_{1}-\ldots-u_{s}^{t} k_{s} ; \mu_{1}^{t}, \ldots, \mu_{r}^{t}\right),\left(b_{j} ; \beta_{j}^{\prime}, \ldots, \beta_{j}^{(r)}\right)_{1, Q}:\left(d_{j}^{\prime}, \delta_{j}^{\prime}\right)_{1, Q^{\prime}} ; \ldots ;\left(d_{j}^{(r)}, \delta_{j}^{(r)}\right)_{1, Q}^{(r)}
\end{array}\right] .
\end{aligned}
$$

Provided that

(i) $R e(v)>0$; the quantities $u_{1}, u_{1}^{(1)}, \ldots, u_{s}^{(1)}, \ldots, u_{s}, u_{1}^{(t)}, \ldots, u_{s}^{(t)}, \mu_{1}, \mu_{1}^{(1)}, \ldots, \mu_{1}^{(t)}, \mu_{r}, \mu_{r}^{(1)}, \ldots, \mu_{r}^{(t)}$ are all positive (some of them may however decrease to zero provided that the resulting integral has a meaning),

(ii) $\operatorname{Re}(\rho)+\sum_{i=1}^{r} \mu_{i} \min _{1 \leq j \leq M^{(i)}}\left[\operatorname{Re}\left(d_{j}^{(i)} / \delta_{j}^{(i)}\right)\right]+1>0$.

\section{B. Fractional integral formula 2}

$$
\begin{aligned}
& I_{z}^{\eta, v}\left\{z^{\rho} \prod_{i=1}^{t}\left(z+\alpha_{i}\right)^{\sigma_{i}} \prod_{j=1}^{s} S_{n_{j}}^{m_{j}}\left[e_{j} z^{u_{j}} \prod_{i=1}^{t}\left(z+\alpha_{i}\right)^{u_{j}^{(i)}}\right]\right. \\
& \left.\times H\left[x_{1} z^{\mu_{1}} \prod_{i=1}^{t}\left(z+\alpha_{i}\right)^{\mu_{1}^{(i)}}, \ldots, x_{r} z^{\mu_{r}} \prod_{i=1}^{t}\left(z+\alpha_{i}\right)^{\mu_{r}^{(i)}}\right]\right\} \\
& =z^{\rho}\left(\alpha_{1}\right)^{\sigma_{1}} \cdots\left(\alpha_{t}\right)^{\sigma_{t}} \sum_{g_{1}=0}^{\infty} \cdots \sum_{g_{t}=0}^{\infty} \sum_{k_{1}=0}^{\left[n_{1} / m_{1}\right]} \cdots \sum_{k_{s}=0}^{\left[n_{s} / m_{s}\right]} \frac{\left(-n_{1}\right)_{m_{1}} k_{1}}{k_{1} ! g_{1} !} A_{n_{1}, k_{1}}^{(1)} \cdots \\
& \times \frac{\left(-n_{s}\right)_{m_{s} k_{s}}}{k_{s} ! g_{s} !} A_{n_{s}, k_{s}}^{(s)} e_{1}^{k_{1}} \cdots e_{s}^{k_{s}}\left(\alpha_{1}\right)^{\left[u_{1}^{(1)} k_{1}+\cdots+u_{s}^{(1)} k_{s}-g_{1}\right]} \cdots\left(\alpha_{t}\right)^{\left[u_{1}^{(t)} k_{1}+\cdots+u_{s}^{(t)} k_{s}-g_{t}\right]} \\
& \times z^{\left[u_{1} k_{1}+\cdots+u_{s} k_{s}+g_{1}+\cdots+g_{t}\right]} \quad H_{P+(t+1), Q+(t+1): P^{\prime}, Q^{\prime} ; \ldots ; P^{(r)}, Q^{(r)}}^{0, N+(t+1): M^{\prime}, N^{\prime} ; \ldots ; M^{(r)}, N^{(r)}}
\end{aligned}
$$

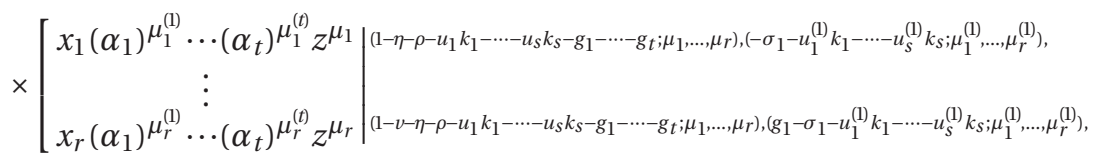

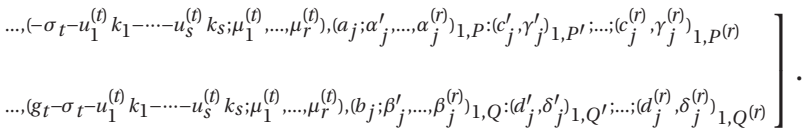

Provided that

(i) $\eta>0$; the quantities $u_{1}, u_{1}^{(1)}, \ldots, u_{s}^{(1)}, \ldots, u_{s}, u_{1}^{(t)}, \ldots, u_{s}^{(t)}, \mu_{1}, \mu_{1}^{(1)}, \ldots, \mu_{1}^{(t)}, \mu_{r}, \mu_{r}^{(1)}, \ldots, \mu_{r}^{(t)}$ are all positive (some of them may however decrease to zero provided that the resulting integral has a meaning), 
(ii) $\operatorname{Re}(\rho)+\sum_{i=1}^{r} \mu_{i} \min _{1 \leq j \leq M^{(i)}}\left[\operatorname{Re}\left(d_{j}^{(i)} / \delta_{j}^{(i)}\right)\right]+\eta>0$.

Proof. In order to prove (2.1), we first express a general class of polynomials in series form given by (1.3) and multivariate $H$-function in terms of Mellin-Barnes type of contour integrals and interchanging the order of summations, integration and taking the fractional integral operator inside, which is permissible under the stated conditions. Now, using binomial expansion along with the use of the known formula (1.1) and interpreting the multiple Mellin-Barnes contour integral so obtained in terms of $H$-function, we easily arrive at the desired formula (2.1).

Also, using the same method adopted in the proof of the result (2.1) and making use of the formula [12, eq.(2.10)]

$$
I_{z}^{\eta, \nu}\left[z^{\lambda}\right]=\frac{\Gamma(\lambda+\eta)}{\Gamma(\lambda+\eta+v)} z^{\lambda}, \quad \operatorname{Re}(\lambda)>-\eta
$$

we can prove the result (2.4).

\section{Special cases}

3.A. If we put $t=2$ and $s=2$ in our integral formula (2.1), it reduces to the known result recently obtained by Gaira and Dhami [12, p.2, eq.(2.1)].

3.B. On taking $t=2$ and $s=2$ our integral formula (2.4) reduces to another known result obtained by Gaira and Dhami [12, p.5, eq.(2.9)].

3.C. Letting $t=1$ and $s=1$ in our result (2.4), it leads to a known result given by Gupta and Agarwal [9].

3.D. Particularly, when we substitute $t=1$ and $n_{j}=0(j=0,1, \ldots, s)$ in our integral formula (2.3), we arrive on the result obtained by Srivastava et al. [7].

3.E. If we put $m_{j}=n_{j}=k_{j}=0$ (for $j=2,3, \ldots, s$ ) and $t=1$ in our integral formula (2.1), we can derive another result obtained by Gupta et al. [9].

\section{Acknowledgement}

The authors are grateful to Professor H. M. Srivastava, University of Victoria, Canada for his kind help and valuable suggestions in the preparation of this paper. The authors are also thankful to the worthy referee for his valuable suggestions in the improvement of the paper.

\section{References}

[1] B. Ross, Fractional calculus and its applications, Lecture notes in Math., Springer-Verlag, New York, 457(1975).

[2] H. M. Srivastava, A contour integral involving Fox's H-function, Indian J. Math. 14(1972), 1-6. 
[3] H. M. Srivastava and R. Panda, Some bilateral generating function for a class of generalized hypergeometric polynomials, J. Reine Angew. Math. 283/284(1976), 265-276.

[4] H. M. Srivastave, K. C. Gupta and S. P. Goyal, The $H$-Functions of One and Two Variables with Applications, South Asian Publishers, New Delhi, 1982.

[5] H. M. Srivastava and S. P. Goyal, Fractional derivative of the H-function of several variables, J. Math. Anal. Appl. 112(1985), 641-651.

[6] H. M. Srivastava, R. S. Chandel and P. K. Vishwakarma, Fractional derivative of certain generalized hypergeometric functions of several variables, J. Math. Anal. Appl. 184(1994), 560-572.

[7] H. M. Srivastava, Fractional calculus and its applications, Cubo, Mat. Ed. 5(2003), 33-48.

[8] K. B. Oldham and J. Spanier, The Fractional Calculus, Academic Press, New York/London, 1974.

[9] K. C. Gupta and S. M. Agrawal, Fractional integral formulae involving a general class of polynomials and the multivariable H-function, Proc. Indian Acad. Sci. (Math. Sci.), 99(1989), 169-173.

[10] K. C. Kupta and R. C. Soni, A study of H-function of one and several variables, J. Rajasthan Acad. Phys. Sci. 1(2002), 89-94.

[11] M. Saigo and R. K. Raina, Fractional calculus operators associated with a general class of polynomials, Fukuoka Univ. Sci. Reports 18(1988), 15-22.

[12] M. K. Gaira and H. S. Dhami, Fractional integral formulae involving the product of a general class of polynomials and the multivariate H-function, IMA Preprint Series, 1999(2004). URL: http://www.ima.umn.edu

[13] M.-P. Chen, H. M. Srivastava and C.-S. Yu, Some operators of fractional calculus and their applications involving a new class of analytic function, Appl. Math. Comt. 91(1998), 285-296.

[14] N. P. Singh and H. M. Srivastava, The integration of certain product of the multivariable H-function with a general class of polynomials, Rendicontidel Circolo Mathematico di Palermi, Ser. II 32(1983), 152-187.

[15] R. C. Soni and Deepika Singh, Certain fractional derivatives formulae involving the product of general class of polynomials and multivariable H-functions, Proc. Indian Acad. Sci. (Math. Sci.) 112(2002), 551-562.

[16] S.-D. Lin, S.-T. Tu and H. M. Srivastava, New generating function for a class of generalized Hermite polynomials, J. Math. Anal. Appl. 261(2001), 479-496.

[17] S.-D. Lin, J.-C. Shyu, K. Nishimoto and H. M. Srivastava, Explicit solutions of some general families of ordinary and partial differential equations associated with Bessel equation, by means of fractional calculus, J. Fract Calc. 25(2004), 33-45.

Department of Mathematics, University of Rajasthan, Jaipur-302004, India.

Department of Mathematics and Statistics, Banasthali Vidyapith, AIM \& ACT, Jaipur-302001, India.

E-mail: sharedpandey@yahoo.co.in 